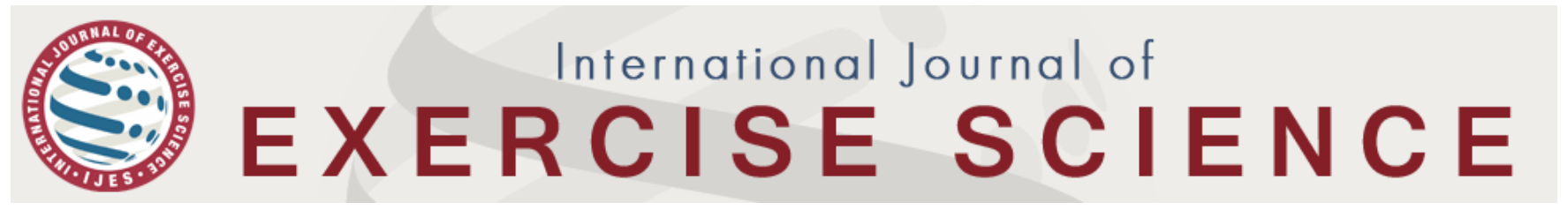

\title{
COVID-19 Lockdowns: Exacerbating the Silent Pandemic
}

\section{DAVID GOMEZ ${ }^{\dagger 1}$, ERIC V. NEUFELD ${ }^{\dagger 1,2}$, JAMES W. HICKS $\$ 3$, and BRETT A. DOLEZAL ${ }^{1}$}

${ }^{1}$ Airway and Exercise Physiology Research Laboratory, David Geffen School of Medicine, University of California-Los Angeles, Los Angeles, CA, USA; ${ }^{2}$ Donald and Barbara Zucker School of Medicine at Hofstra/Northwell, Hofstra University, Hempstead, NY, USA; ${ }^{3}$ Center for Exercise Medicine and Sport Sciences, University of California-Irvine, Irvine, CA, USA

†Denotes graduate student author, ‡Denotes professional author

\section{ABSTRACT}

International Journal of Exercise Science 14(3): 1256-1260, 2021. The global medical community has exalted the vaccine as the champion solution to end the violent toll inflicted by COVID-19. While the role of vaccines cannot be undervalued in wide-scale intervention, presenting them as the sole solution exonerates individuals of the importance of taking ownership over their lifestyle choices. This editorial focuses on the importance of physical activity as a crucial component of COVID-19 prevention programs and a long-term investment against chronic diseases.

KEY WORDS: Sedentary behavior, physical activity, exercise, infectious diseases, chronic diseases

The COVID-19 pandemic brought life to a screeching halt. Efforts designed to limit transmission such as mask mandates and social distancing quickly shifted their gaze to the very movement of people, closing among most businesses - recreational facilities such as gyms, parks, and beaches. This precautionary, near-global house arrest exacerbated a pre-existing, often forgotten pandemic: physical inactivity. The World Health Organization estimates that 5 million deaths could be avoided annually if the global population was more active (20). That surpasses the total number of registered COVID-19 deaths to date by half a million (19). Despite the wealth of scientific evidence emphasizing the necessity of exercise to maintain health in people of all ages, exercise referral

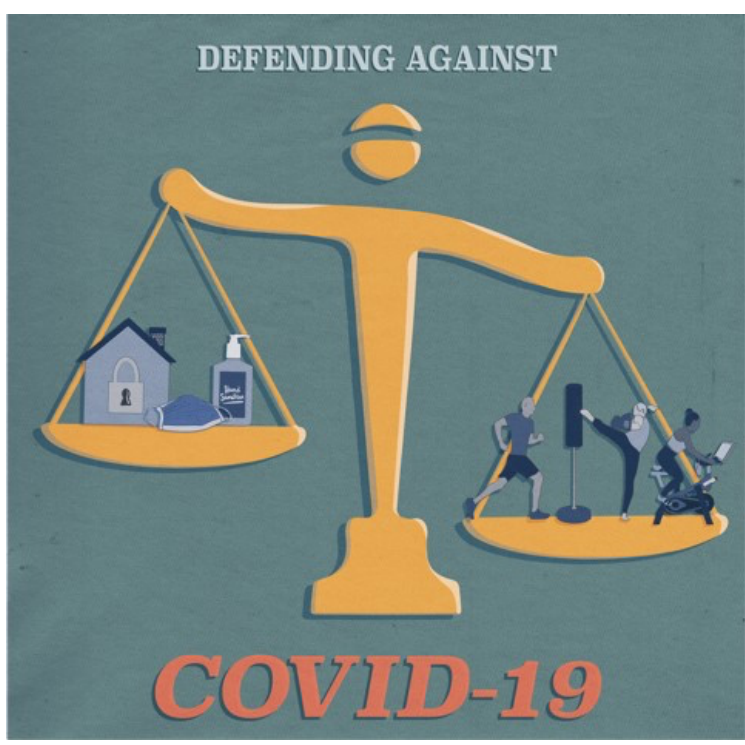
by the medical community is lacking in most places around the world. The link between growing inactivity and the number of hospitalizations and deaths of COVID-19 
patients (14) makes it increasingly inexcusable to view these two pandemics as separate. The pandemic enabled physical inactivity and all of its sequelae such as metabolic syndrome and behavioral health problems. Public health measures over the past 18 months have been almost unilaterally aimed at preventing contact with the new coronavirus. While this is inarguably important, COVID-19 remains highly contagious and it is now necessary to recognize the uncomfortable truth that this approach was taken at the expense of optimizing individuals' immune systems to rid the virus after nearly inevitable contact.

The medical community has overwhelmingly emphasized the role of vaccines as the one key solution to halt the violent toll the virus has taken on the global population. While the role of vaccines cannot be undervalued, presenting them as the sole solution exonerates individuals of the importance of the health of their own lifestyles. According to a recent paper published in the Journal of the American Heart Association, 30\% of COVID-19 hospitalizations occurring in November 2020 were attributable to obesity, $26 \%$ to hypertension, $21 \%$ to diabetes, and $12 \%$ to heart failure. Their model predicted that almost two-thirds of the hospitalizations might have been prevented if these comorbidities that affect the immune system had not been present (11). In other words, the number of infections may have been the same, but a stronger immune system brought on by habitual exercise might have been able to fight the infection without needing hospitalization. Beyond the myriad benefits of exercise, an active lifestyle proves even more beneficial and far more important in our current global state: it is an effective preventive tool for most of the pre-existing chronic conditions that predict COVID19 hospitalizations $(6,15,23)$, improves immune system response $(10,17,1)$, and may also enhance vaccine-induced antibody production (2). Regular moderate-to-vigorous physical activity has been shown to increase the body's antibody response to vaccination as well as lower the risk of community-acquired infection by $31 \%$ and risk of infectious-disease mortality by $37 \%$ (2). On the molecular level, physical activity may help protect against acute respiratory distress syndrome (ARDS) and multiorgan failure-severe complications of COVID-19-by upregulating the potent antioxidant enzyme extracellular superoxide dismutase (EcSOD) $(8,21,22)$. However, this is only the tip of the iceberg. The amount of existing research implicating physical inactivity as a key contributor to many chronic diseases is overwhelming.

The COVID-19 pandemic has made it glaringly obvious that we must break the habits and mindset of sedentary behavior and underscore the importance of physical activity. Although scientists have posited that exercising safely (i.e., isolated and outdoors or indoors with social distancing and mask wearing) is a valuable way to strengthen our immune system and protect ourselves from our unseen enemy $(4,5)$, it is worrisome how many people-aware of this information-still spend more time looking at screens rather than staying physically active. The pandemic-induced social isolation decreased physical activity $(g=-0.913)$, increased sitting time $(g=0.698)$, and screen time $(g=0.653)$ among those who were already active before the pandemic. Furthermore, sitting and screen time also increased among the inactive population $(g=0.565, g=0.589)$. Beyond the physical consequences, the disruption of normal lifestyles has strongly impacted mental health with greater levels of stress, sleep disorders, eating disorders, anxiety, panic attacks, and depression (9). These consequences are exacerbated in communities with less resources as unequal access to healthcare and education, 
financial insecurity, and discrimination have added to the daily strain of individuals. Nevertheless, exercise helps combat the psychological impact of prolonged periods of social isolation, reducing feelings of anxiety, depression, and stress $(3,10,16,18,24)$.

We are not out of the woods yet. The fourth wave of the pandemic, quarterbacked by the highly infectious Delta variant of the virus, underlines the importance of wide spread vaccination to reduce the number of deadly cases. Continued virus mutations prolong the return to 'normalcy' and require an expansion beyond (though still including) an emphasis on limiting the spread via vaccination, social distancing, and mask mandates. Much of the world has reactively plugged leaks to mitigate short term collateral, severely undermining complementary efforts to mitigate potentially disastrous long-term damage. For example, social distancing mandates that have helped save lives have in other cases exacerbated the consequences of physical inactivity. Habitual exercise is a long-term investment on an individual and national scale, as it helps prevent chronic illnesses, as well as devastating economic impacts - physical inactivity is associated with $1.5-3 \%$ of total direct healthcare cost in developed countries (12). The role of exercise as medicine is not a new concept, as it has shown beneficial effects on 23 diseases or health conditions (13), but it is largely omitted from regulatory integration. The obvious urgency of COVID-19 presents a unique opportunity to address both pandemics simultaneously by shifting public mindsets, government efforts and medical training from palliative to preventative medicine. It is time to encourage people to go out safely and exercise regularly. We have this powerful and affordable "drug" with very few side effects already at our disposal. With international guidelines recommending 150-300 minutes of moderate-intensity activity per week in order to reap the health benefits of exercise (7), that include improved immune function, reduced risks and improved outcomes for a variety of cancers, enhanced cognition and memory, improvements in mental health, higher overall quality of life and promotion of healthy aging. The numerous benefits beg the question: "Have you been exercising enough?"

\section{REFERENCES}

1. Campbell JP, Turner JE. Debunking the myth of exercise-induced immune suppression: Redefining the impact of exercise on immunological health across the lifespan. Front Immunol 9: 648, 2018.

2. Chastin SFM, Abaraogu U, Bourgois JG, Dall PM, Darnborough J, Duncan E, et al. Effects of regular physical activity on the immune system, vaccination and risk of community-acquired infectious disease in the general population: Systematic review and meta-analysis. Sports Med 51(8): 1673-1686, 2021.

3. Chekroud SR, Gueorguieva R, Zheutlin AB, Paulus M, Krumholz HM, Krystal JH, et al. Association between physical exercise and mental health in 1.2 million individuals in the USA between 2011 and 2015: A cross-sectional study. Lancet Psychiatry 5(9): 739-746, 2018.

4. Hopkins SR, Dominelli PB, Davis CK, Guenette JA, Luks AM, Molgat-Seon Y, et al. Face masks and the cardiorespiratory response to physical activity in health and disease. Ann Am Thorac Soc 18(3): :399-407, 2021

5. Howard J, Huang A, Li Z, Tufekci Z, Zdimal V, van der Westhuizen H-M, et al. An evidence review of face masks against COVID-19. Proc Natl Acad Sci 118(4): e2014564118, 2021. 
6. Huang I, Lim MA, Pranata R. Diabetes mellitus is associated with increased mortality and severity of disease in COVID-19 pneumonia - A systematic review, meta-analysis, and meta-regression. Diabetes Metab Syndr 14(4): 395403, 2020.

7. Kara E. Coronavirus (COVID-19) pandemic: Immunity and exercise intervention. Int J Appl Exerc Physiol 9(10): 97-104, 2020.

8. Martin SA, Pence BD, Woods JA. Exercise and respiratory tract viral infections. Exerc Sport Sci Rev 37(4): 157$164,2009$.

9. Meyer J, McDowell C, Lansing J, Brower C, Smith L, Tully M, et al. Changes in physical activity and sedentary behavior in response to COVID-19 and their associations with mental health in 3052 US adults. Int J Environ Res Public Health 17(18): 6469, 2020.

10. Nieman DC, Wentz LM. The compelling link between physical activity and the body's defense system. J Sport Health Sci 8(3): 201-217, 2019.

11. O'Hearn M, Liu J, Cudhea F, Micha R, Mozaffarian D. Coronavirus disease 2019 hospitalizations attributable to cardiometabolic conditions in the United States: A comparative risk assessment analysis. J Am Heart Assoc 10(5): e019259, 2021.

12. Oldridge NB. Economic burden of physical inactivity: Healthcare costs associated with cardiovascular disease. Eur J Cardiovasc Prev Rehabil 15(2): 130-139, 2008.

13. Pratt M, Norris J, Lobelo F, Roux L, Wang G. The cost of physical inactivity: Moving into the 21st century. Br J Sports Med 48(3): 171-173, 2014.

14. Sallis R, Young DR, Tartof SY, Sallis JF, Sall J, Li Q, et al. Physical inactivity is associated with a higher risk for severe COVID-19 outcomes: A study in 48440 adult patients. Br J Sports Med 55(19): 1099-1105, 2021.

15. Sharman JE, La Gerche A, Coombes JS. Exercise and cardiovascular risk in patients with hypertension. Am J Hypertens 28(2): 147-158, 2015.

16. Shaphe MA, Chahal A. Relation of physical activity with the depression: A short review. J Lifestyle Med 10(1): $1-6,2020$.

17. Silverman MN, Deuster PA. Biological mechanisms underlying the role of physical fitness in health and resilience. Interface Focus 4(5): 20140040, 2014.

18. Stubbs B, Vancampfort D, Rosenbaum S, Firth J, Cosco T, Veronese N, et al. An examination of the anxiolytic effects of exercise for people with anxiety and stress-related disorders: A meta-analysis. Psychiatry Res 249: 102108, 2017.

19. World Health Organization. Coronavirus Disease (COVID-19) Pandemic. Retrieved from: https://covid19.who.int/; 2021.

20. World Health Organization. Modifiable risk factors for non-communicable diseases. Retrieved from: https://www.who.int/news-room/fact-sheets/detail/physical-activity/; 2021.

21. Yan Z, Spaulding HR. Extracellular superoxide dismutase, a molecular transducer of health benefits of exercise. Redox Biol 32: 10158, 2020. 
Int J Exerc Sci 14(3): 1256-1260, 2021

22. Yang F, Shi S, Zhu J, Shi J, Dai K, Chen X. Analysis of 92 deceased patients with COVID-19. J Med Virol 92(11): 2511-2515, 2020.

23. Yang Z, Scott CA, Mao C, Tang J, Farmer AJ. Resistance exercise versus aerobic exercise for type 2 diabetes: A systematic review and meta-analysis. Sports Med 44(4): 487-499, 2014.

24. Zhai L, Zhang Y, Zhang D. Sedentary behavior and the risk of depression: A meta-analysis. Br J Sports Med 49(11): 705-709, 2015. 